\title{
Identification of ECG Arrhythmias Using Phase Space Reconstruction
}

\author{
Felice M. Roberts ${ }^{1}$, Richard J. Povinelli ${ }^{1}$, and Kristina M. Ropella ${ }^{2}$ \\ ${ }^{1}$ Department of Electrical and Computer Engineering, Marquette University, Milwaukee, WI \\ \{felice.roberts, richard.povinelli\}@mu.edu \\ ${ }^{2}$ Department of Biomedical Engineering, Marquette University, Milwaukee, WI \\ k.ropella@marquette.edu
}

\begin{abstract}
Changes in the normal rhythmicity of a human heart may result in different cardiac arrhythmias, which may be immediately fatal or cause irreparable damage to the heart when sustained over long periods of time. The ability to automatically identify arrhythmias from ECG recordings is important for clinical diagnosis and treatment, as well as, for understanding the electrophysiological mechanisms of the arrhythmias. This paper proposes a novel approach to efficiently and accurately identify normal sinus rhythm and various ventricular arrhythmias through a combination of phase space reconstruction and machine learning techniques. Data was recorded from patients experiencing spontaneous arrhythmia, as well as, induced arrhythmia. The phase space attractors of the different rhythms were learned from both inter- and intra-patient arrhythmic episodes. Out-of-sample ECG rhythm recordings were classified using the learned attractor probability distributions with an overall accuracy of $83.0 \%$.
\end{abstract}

\section{Introduction}

Thousands of deaths occur daily due to ventricular fibrillation (VF)[1]. Ventricular fibrillation is a disorganized, irregular heart rhythm that renders the heart incapable of pumping blood. It is fatal within minutes unless externally terminated by the passage of a large electrical current through the heart muscle. Automatic defibrillators, both internal and external to the body, have proven to be the only therapy for thousands of individuals whom experience ventricular arrhythmia. There is evidence [2] to suggest that the sooner electronic therapy is delivered following the onset of VF, the greater the success of terminating the arrhythmia, and thus, the greater the chance of survival. Defibrillators are required to classify a cardiac rhythm as life threatening before the device can deliver shock therapy; the patient is usually unconscious. Because of the hemodynamic consequences (i.e., the heart ceases to contract, thus no blood flows through the body) that accompany the onset of lethal VF, a preventive approach for treating ventricular arrhythmia is preferable, such as low-energy shock, pacing regimens and/or drug administration to prevent the fatal arrhythmia from occurring in the first place. Furthermore, there is evidence [3] to suggest that high-energy shocks delivered during lethal arrhythmia may be harmful to the myocardium. Thus, the ability to quickly identify and/or predict the impending onset of VF is highly desirable and may increase the alternate therapies available to treat an individual prone to VF. 
Many of the current algorithms differentiate ventricular arrhythmias using classical signal processing techniques, i.e., threshold crossing intervals, autocorrelation, VFfilter, spectral analysis [4], time-frequency distributions [5], coherence analysis [6], and heart rate variability $[7,8]$. In order to improve frequency resolution and minimize spectral leakage, these algorithms need five or more seconds of data when classifying the rhythms. This paper proposes that phase space embedding [9] combined with data mining techniques [10] can learn and accurately characterize chaotic attractors for the different ventricular tachyarrhythmias in short data intervals. Others who have used phase space techniques to study physiological changes in the heart include Bettermann and VanLeeuwen [11], who demonstrated that the changes in heart beat complexity between sleeping and waking states were not a simple function of the heart beat intervals, rather the changes in heart beat complexity were related to the existence of dynamic phases in heart period dynamics.

In this study, signals from two leads of a normal twelve lead ECG recording [12, 13] are transformed into a reconstructed state space, also called phase space. Attractors are learned for each of the following rhythms: sinus rhythm (SR), monomorphic ventricular tachycardia (MVT), polymorphic ventricular tachycardia (PVT), and ventricular fibrillation. A neural net is used to learn the attractors using features formed from the two-dimensional reconstructed phase space. Attractors are learned and tested from inter- and intra-patient data.

\subsection{ECG Recording Overview}

An ECG recording is a measure of the electrical activity of the heart from electrodes placed at specific locations on the torso. A synthesized surface recording of one heartbeat during SR can be seen in Figure 1. The cardiac cycle can be divided into several features. The main features are the $\mathrm{P}$ wave, PR interval, QRS complex, $\mathrm{Q}$ wave, ST segment, and T wave. Each of these components represents the electrical activity in the heart during a portion of the heartbeat [14].

- The P wave represents the depolarization of the atria.

- The PR interval represents the time of conduction from the onset of atrial activation to the onset of ventricular activation through the bundle of His.

- The QRS complex is a naming convention for the portion of the waveform representing the ventricular activation and completion.

- The ST segment serves as the isoelectric line from which amplitudes of other waveforms are measured, and also is important in identifying pathologies, such as myocardial infarctions (elevations) and ischemia (depressions).

- The T wave represents ventricular depolarization.

Recordings seen at different lead locations on the body may exhibit different morphological characteristics. Differences in the ECG recordings from one lead to another are a result of the electrodes being placed at different positions with respect to the heart. Thus the projection of the electrical potential at a point near the sinoatrial node would differ from that seen by an electrode near the atrioventricular node. Differences 
in recordings from one person to another may be due to the difference in the size of the hearts, the orientation of the heart in the body, exact lead location, and the healthiness of the heart itself.

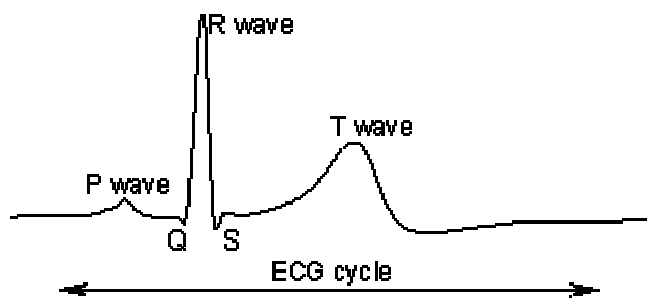

Fig. 1. Synthesized ECG recording for one heartbeat

\section{Methods}

\section{$2.1 \quad$ Recordings}

Simultaneous recordings of surface leads II and V1 of a normal 12 lead ECG [12, 13] were obtained from six patients using an electrophysiological recorder. These patients exhibited sustained monomorphic ventricular tachycardia, polymorphic ventricular tachycardia, ventricular fibrillation and/or any combination of these rhythms during electrophysiological testing (EP) and/or automatic implantable cardioverter/defribrillator (AICD) implantation. None of the data was from healthy patients.

Two independent observers classified the ECG recordings as one of the following rhythms: VF, PVT, MVT, and SR. The criteria for classifying of the different rhythms were [15-17]:

- $\quad$ VF was defined by undulations that were irregular in timing and morphology without discrete QRS complexes, ST segments, or T waves with cycle length $<200$ msec.

- PVT was defined as ventricular tachycardia having variable QRS morphology but with discrete QRS complexes with cycle length $<400 \mathrm{msec}$.

- MVT was defined as ventricular tachycardia having a constant QRS morphology with cycle length $<600$ msec.

- SR was defined by rhythms exhibiting P waves, QRS complexes, ST segments, and $\mathrm{T}$ waves with no aberrant morphology interspersed in the data interval.

Ventricular tachycardia is most commonly associated in patients with coronary artery disease and prior myocardial infarctions. Patients with dilated cardiomyopathies, arrythmogenic right ventricular dysplasia, congenital heart disease, hypertrophic cardiomyopathy, or mitral valve prolapses experience VT. Infrequently VT occurs in patients without identifiable heart abnormalities[18]. Ventricular fibrillation occurs primarily in patients with transient or permanent conduction block. Patients experi- 
ence VF under a variety of conditions, including: 1) electrically induced by a lowintensity stimulus delivered while the ventricles are repolarizing; 2) electrically induced by a burst (approximately 1 second duration) of $60 \mathrm{~Hz}$ AC current; 3) spontaneously induced due to ischemia leading to a conduction block; 4) reperfusioninduced; and 5) electrically induced by high-intensity electric shocks[16].

Examples of the different rhythm morphologies can be seen in Fig. 2.

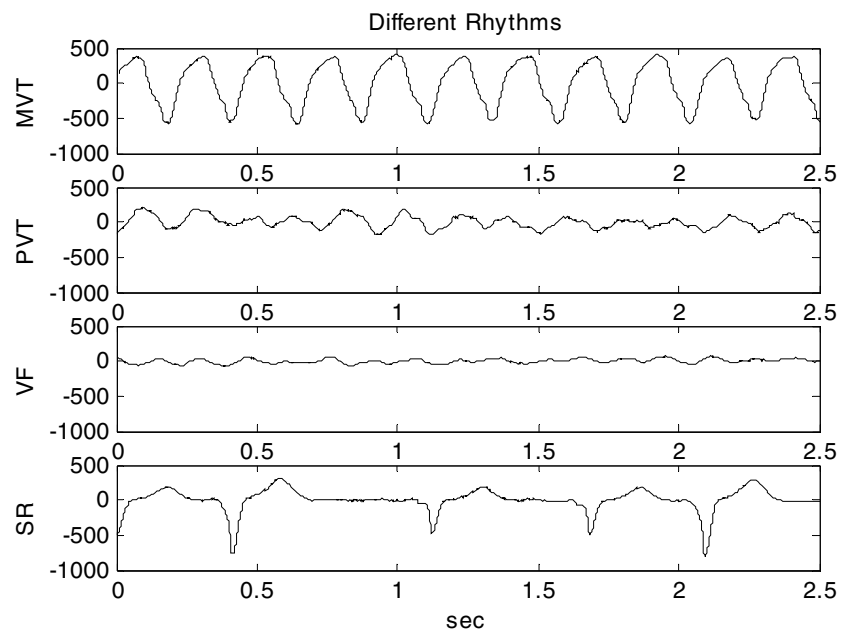

Fig. 2. Recording for individual examples of rhythm morphologies: monomorphic ventricular tachycardia (MVT), polymorphic ventricular tachycardia (PVT), ventricular fibrillation (VF), and sinus rhythm (SR)

\subsection{Preprocessing}

Data were antialiased filtered with a cutoff frequency of $200 \mathrm{~Hz}$ and subsequently digitized at 1,200 Hz. Up to 60 seconds of continuous data were digitized for each rhythm. In this study, the data was divided into 2.5-second contiguous intervals of MVT, PVT, VF or SR rhythms. The data were zero-meaned prior to further analysis.

\subsection{Feature Identification}

A two-dimensional phase space was constructed using the II and V1 ECG recordings. Figure 3 illustrates the generated phase space.

Each rhythm is attracted to a different subset of the phase space. This subset of the phase space is the attractor for that particular rhythm. Visually, one can differentiate the rhythm attractors in Fig. 3. However, for an automatic defibrillator to automatically classify rhythms, features must be determined that define each attractor. These features were generated using the following method. 

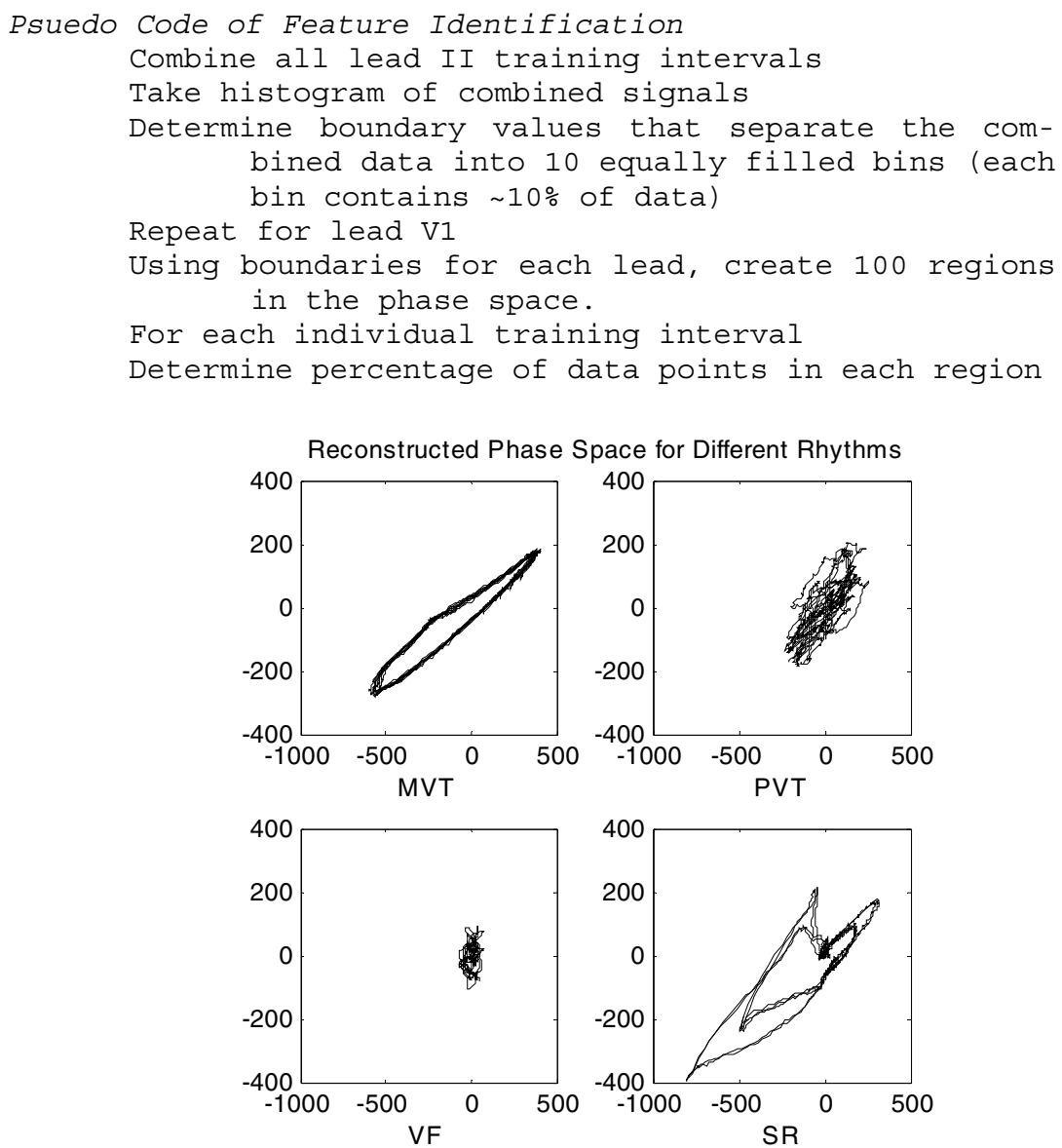

Fig. 3. Generated two-dimensional phase space for examples of MVT, PVT, VF, and SR. Notice that the different rhythms fill a different subset of the phase space

An example of the regions subdividing the phase space for an SR rhythm can be seen in Fig. 4.

\subsection{Attractor Learning}

The attractors were learned using neural networks with 100 inputs, one output, and two hidden layers. The first and second hidden layers consisted of 10 and 3 neurons with tan-sigmoid transfer functions, respectively. The output layer was a log-sigmoid neuron. The neural net was learned using the Levenberg-Marquardt algorithm in MATLAB. The inputs to the neural networks were the percentage of data points in each feature bin described in previously. Leave-one-out cross-validation [19] was used in the training and testing of the neural networks. Given an indexed data set 
$\left\{d_{i}: i=1, \ldots, n\right\}$ containing $n$ elements, $n$ training/testing runs are performed. For the $j^{\text {th }}$ run, the test set is $\left\{d_{j}\right\}$ and the training set is $\left\{d_{i}: \forall i \neq j\right\}$.

Individual neural networks were used to classify each rhythm. The output of the neural net was rounded, in order that 1 classified the input data as the specific rhythm, 0 classified it as some other rhythm. For a patient exhibiting two different morphologies, two neural networks would be trained and tested to classify the ECG intervals. An example of the classifier architecture for Patient 2 can be seen in Fig. 5. To be a legitimate classification, only one neural network can classify the signal.

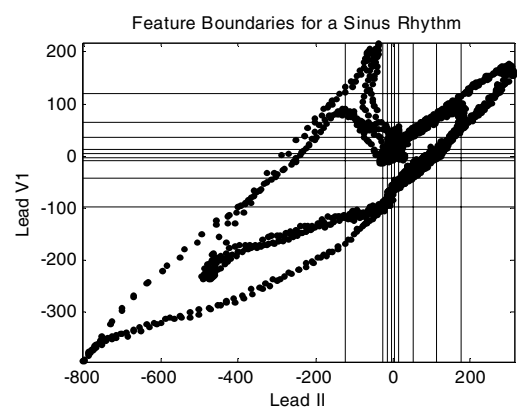

Fig. 4. Example of feature bin boundaries for a 2.5 second recording of sinus rhythm

\subsection{Comparative Analysis}

We compare our new method against three others. The first comparison is to a method based on the Lempel-Ziv complexity measure. The second comparison is to a method based on heart rate. The third comparison is to two independent human expert observers.

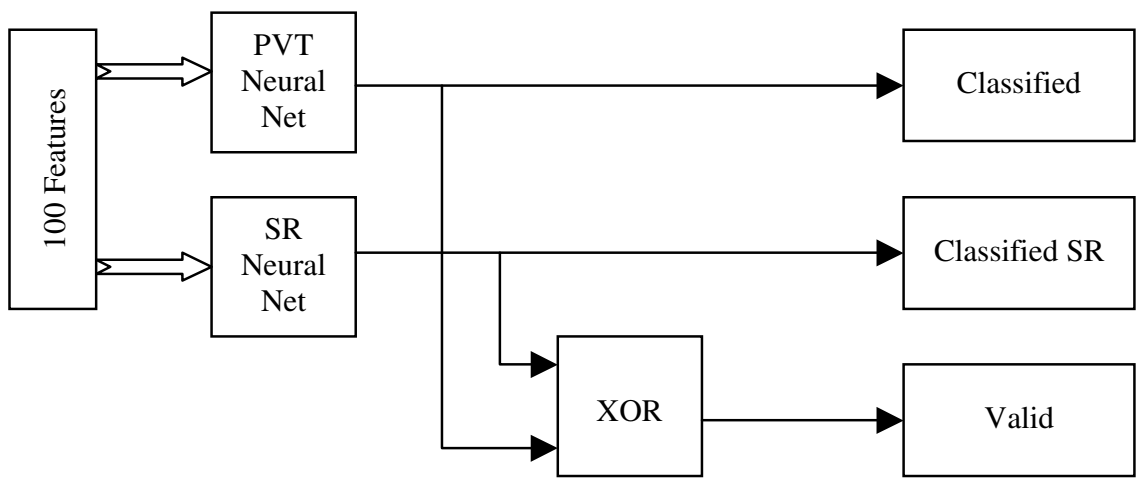

Fig. 5. Classifier architecture. The number of rhythm neural nets corresponds to the number of rhythms for a particular set of data. For sets of data with more than two rhythms to classify the $\mathrm{XOR}$ box is more complicated than a single exclusive OR 
Zhang et al. [20] proposed a method for detecting MVT, VF, and SR using the Lempel-Ziv complexity measure. The complexity measure is a function of the number of patterns found in a string of threshold crossings. For each interval of data, a new threshold was calculated. As with the method proposed in this paper, Zhang's complexity measure does not need to detect the occurrences of beats. They used various interval lengths to determine the minimum amount of data needed to attain $100 \%$ training accuracy; no test accuracy was determined. A seven second interval was found to be the minimum length needed to correctly discriminating the three rhythms. For the rhythms (MVT, VF, and SR), intervals of length two and three seconds achieved training accuracies of $(93.14 \%, 95.10 \%$, and $98.04 \%)$ and $(93.14 \%, 97.55 \%$, and $95.59 \%$ ), respectively. Zhang classified the rhythms using the following cutoff values for the complexity measures:

- $\quad \mathrm{SR}$ - for complexity measures less than 0.150

- $\quad$ MVT - for complexity measures between 0.150 and 0.486

- $\quad \mathrm{VF}$ - for complexity measures greater than 0.486 .

Heart rate is used in many AICDs to discriminate one rhythm from another. Medtronic, Inc. a commercial maker of AICDs uses rate detection zones and different counts to detect and classify tachyarrhythmias [17]. AICDs count the number of beats in each detection zone, if a specified number of beats are within a particular zone without a SR rhythm beat being detected, the interval is marked as a tachyarrhythmia. Since the data intervals used are only two and half seconds long, there are not enough beats to be counted, so only the heart rate is used to classify the rhythm intervals. For each individual interval, thresholds for marking a new beat were set to $60 \%$ of the maximum amplitude of that interval.

\section{Results}

\subsection{Data}

Six patients comprised the study population. The heart rhythms exhibited by the six patients can be seen in Table 1. Four of the patients exhibited different combinations of two or three types of rhythms. The last two patients exhibited all four types of rhythms. Two independent observers performed the original rhythm classification.

Table 1. Patient and number of $2.5 \mathrm{~s}$ rhythm intervals experienced

\begin{tabular}{ccccc}
\hline Patient & MVT & PVT & VF & SR \\
\hline 1 & & & 23 & 27 \\
2 & & 6 & 12 & \\
3 & & 23 & & 30 \\
4 & 15 & 8 & 4 & \\
5 & 15 & 8 & 2 & 33 \\
6 & 20 & 6 & 5 & 34 \\
\hline
\end{tabular}


Overall inter-observer agreement for rhythm classification was $80.7 \%$. The two observers conferred to reach consensus on the classification of the remaining $19.3 \%$. The intervals used in this study were not meticulously selected to have comparable amplitudes, waveforms, and heart rates. The intervals were selected blindly from rhythms classified by the two observers.

\subsection{Intra-patient Classification}

For each patient, classifiers were created for each rhythm interval. The neural nets in the classifiers were able to learn the training data within approximately 20 epochs with $100 \%$ accuracy, with leave-one-out cross-validation. For the training data, the classifiers accurately identified rhythm type from $69.8 \%$ to $83.3 \%$ of the time with an overall average accuracy of $77.1 \%$. The accuracy for each patient's classifier is listed in Table 2. Each classifier had four possible outputs:

- Correctly Classified - 2.5-second rhythm interval was classified correctly.

- Incorrectly Classified - 2.5-second rhythm interval was classified as a different rhythm.

- Undetermined (no classification) - 2.5-second rhythm interval was not classified.

- Undetermined (two classifications) - 2.5-second rhythm interval was classified as two rhythms (It should be noted that no rhythm interval was classified as more than two rhythms.)

Table 2. Intra-patient classifier accuracy

\begin{tabular}{cccccc}
\hline Patient & $\begin{array}{c}\text { Correctly } \\
\text { Classified }\end{array}$ & $\begin{array}{c}\text { Incorrectly } \\
\text { Classified }\end{array}$ & $\begin{array}{c}\text { Undetermined } \\
\text { (No } \\
\text { classification) }\end{array}$ & $\begin{array}{c}\text { Undetermined } \\
\text { (2 classifica- } \\
\text { tions) }\end{array}$ & $\begin{array}{c}\text { Percent } \\
\text { Accuracy }\end{array}$ \\
\hline 1 & 41 & 1 & 2 & 6 & $82.0 \%$ \\
2 & 15 & 0 & 2 & 1 & $83.3 \%$ \\
3 & 37 & 3 & 8 & 5 & $69.8 \%$ \\
4 & 21 & 2 & 1 & 3 & $75.0 \%$ \\
5 & 44 & 5 & 3 & 6 & $75.8 \%$ \\
6 & 51 & 1 & 5 & 8 & $78.5 \%$ \\
\hline
\end{tabular}

\subsection{Inter-patient Classification}

All 271 data segments from the six patients were combined and classified. The training data was learned with $100 \%$ accuracy within approximately 30 epochs. Leaveone-out cross-validation was performed. The accuracy of classifying the 271 rhythm intervals was improved compared to the intra-patient classification accuracy. The classification accuracy for the 271 intervals was $83.0 \%$, with the following breakdown of classification: 
- 225 were correctly classified.

- 12 were incorrectly classified.

- 11 were undetermined due to no classification.

- 23 were undetermined due to two or more classifications (only one interval was classified as three separate rhythms).

The confusion matrix for the proposed method is given in Table 3. Recall because of the structure of the proposed classifier, a data interval may be under (no classification) or over (two or more classifications) classified, hence the total classifications in Table 3 is not 271 .

Table 3. Confusion matrix for phase space classification method

\begin{tabular}{lcccccc}
\hline & \multicolumn{3}{c}{ Classified As } & \multicolumn{2}{c}{ Valid } \\
& SR & MVT & PVT & VF & Classification & Accuracy \\
\hline SR & 117 & 1 & 7 & 6 & 109 & $87.9 \%$ \\
MVT & 1 & 47 & 5 & 0 & 42 & $84.0 \%$ \\
PVT & 3 & 4 & 45 & 2 & 39 & $76.5 \%$ \\
VF & 2 & 0 & 6 & 38 & 35 & $76.1 \%$ \\
\hline
\end{tabular}

\subsection{Complexity Measure Inter-patient Classification}

Using the complexity measure algorithm from [20], the complexity measure for each interval was calculated. The distributions of the measures for the different rhythms are shown in Figure 5. It can be seen in the graph that unlike Zhang's training results there is no distinct separation between complexity measures of the different rhythms; nor were the values attained using this data within the same ranges as those determined by Zhang. The results are extremely poor as seen by the accuracies given in Table 4.

Table 4. Confusion matrix for complexity measure classification

\begin{tabular}{lccccc}
\hline & \multicolumn{5}{c}{ Classified As } \\
& SR & MVT & PVT & VF & Accuracy \\
\hline SR & 116 & 8 & 0 & 0 & $93.5 \%$ \\
MVT & 50 & 0 & 0 & 0 & $0 \%$ \\
PVT & 51 & 0 & 0 & 0 & $0 \%$ \\
VF & 38 & 8 & 0 & 0 & $0 \%$ \\
\hline
\end{tabular}




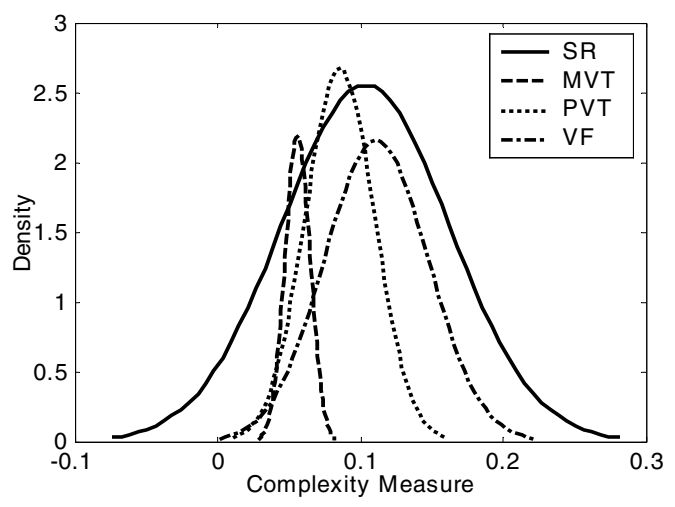

Fig. 6. Complexity measure distribution for the all four rhythm types

\subsection{Heart Rate Inter-patient Classification}

Classification using the heart rate had an overall accuracy of $62 \%$. Misclassifications occurred in all rhythm intervals. The MVT intervals had the worst accuracy. The classification using heart rate can be seen in Table 5.

Table 5. Confusion matrix for heart rate classification

\begin{tabular}{lccccc}
\hline & \multicolumn{5}{c}{ Classified As } \\
& SR & MVT & PVT & VF & Accuracy \\
\hline SR & 83 & 38 & 3 & 0 & $66.9 \%$ \\
MVT & 0 & 11 & 20 & 19 & $22.0 \%$ \\
PVT & 0 & 0 & 40 & 11 & $78.4 \%$ \\
VF & 0 & 1 & 1 & 44 & $95.6 \%$ \\
\hline
\end{tabular}

\section{Discussion}

Ideally, an implantable antitachycardia device should be capable of several modes of therapy including antitachycardia pacing, low-energy cardioversion, and high-energy defibrillation [21-23]. Patients requiring these types of therapy often experience more than one rhythm type. These different arrhythmias may require different therapies. However, for the several modes of therapy to be available in one device, detection algorithms must be able to accurately differentiate among various arrhythmias. The results from this preliminary study are encouraging for developing accurate detection algorithms among the various ventricular tachyarrhythmias. The ability to accurately classify rhythms experienced by individual patients more than $75 \%$ of the time is in close agreement with the classification of trained observers. The classification accuracy across all patients was better for the automated scheme than for the original classification by trained observers. The classification performed using the complexity measures of the rhythms was extremely poor. It is obvious that Zhang's threshold values are not generalizable. Even if new threshold values were determined for our 
data set, their classification method would perform poorly as can be seen in Fig. 6 by the strong overlapping of the classes.

Using the reconstructed phase space to classify out-of-sample ECG recordings performed better than the classification using the heart rate alone. This is due to several reasons. The first and foremost was part of the new algorithm's advantages is the ability to classify ECG rhythms in only 2.5 seconds. Most ICDs require 10 seconds to classify a tachyarrhythmia. Many of the commercial detection algorithms also allow the medical provider to determine templates for the patient's SR. As these were outof-sample intervals no templates could be generated. Thus the detection of heartbeats ranged drastically from one interval to the next. Secondly, as stated previously, the morphology seen in an ECG recording is a function of the healthiness of the heart. And as each of these rhythms was recorded during electrophysiological testing (EP) and/or automatic implantable cardioverter/defribrillator (AICD) implantation, none of these hearts can be considered extremely healthy. Thus individual rhythms greatly vary from one patient to the next. For example during SR, one patient had T-waves whose amplitudes were as large as the QRS. The T-waves were counted as a new heartbeat, thus doubling the calculated heart rate. Finally, even though the data was zero-meaned linear trends were not removed from the intervals, thus fewer beats were counted.

Although the proposed method was accurate $83 \%$ of the time, if used in AICDs in its current form, the misclassification of SR and MVT as VF could cause a patient to receive an unnecessary defibrillation shock which has the possibility of being detrimental to the patient. Some of these false classifications were due to SR intervals in which the maximum amplitude of the signal was not very large, thus the phase space reconstruction of these non-fatal rhythms was very close to that of VF. Further improvement is still needed before these short intervals can be used in commercial applications, such as the development of multi-therapy implantable antitachycardia devices. The high classification accuracy of the proposed method within a short period of time reinforces the author's conjecture that phase space is a valid starting point in the classification of ventricular tachyarrhythmias. Other features will need to be added to the proposed method to improve the classification accuracy for short intervals of data. Further investigations for defining the rhythm attractors will incorporate time-delay and multi-dimensional phase spaces.

Future research into the identification of ventricular tachyarrhythmias may unveil electrophysiological mechanisms responsible for the onset and termination of fibrillatory rhythms. We hypothesize that the patterns of the quasi-periodic [24] attractors of heart rhythms change immediately prior to (within a 10-minute time period) the onset of a serious ventricular arrhythmia. Using these attractors, future research will focus on the transitions from one phase space attractor to another. This may reveal how changes in the attractor space correspond to heart rhythm changes, with the end goal being able to predict the onset of VF, thus improving available therapy and prevention. 


\section{References}

[1] F. X. Witkowski, L. J. Leon, P. A. Penkoske, R. B. Clark, M. L. Spano, W. L. Ditto, and W. R. Giles, "A Method of Visualization of Ventricular Fibrillation: Design of a Cooled Fiberoptically Coupled Image Intensified CCD Data Acquisition System Incorporating Wavelet Shrinkage Based Adaptive Filtering," American Institute of Physics, vol. 8, pp. 94-102, 1998.

[2] E. Manios, G. Fenelon, T. Malacky, A. L. Fo, and P. Brugada, "Life Threatening Ventricular Arrhythmias in Patients with Minimal or no Structural Heart Disease: Experience with the Implantable Cardioverter Defibrillator," available at http://www.heartweb.org/heartweb/0197/icd0003.htm, 1997, cited December 2, 2000

[3] J. A. Stewart, "A More Effective Approach to In-Hospital Defibrillation," Journal of Cardiovascular Nursing, vol. 10, pp. 37-46, 1996.

[4] R. H. Clayton, A. Murray, and R. W. F. Campbell, "Comparison of Four Techniques for Recognition of Ventricular Fibrillation from the surface ECG," Medical and Biological Engineering and Computing, vol. 31, pp. 111-117, 1993.

[5] V. X. Afonso and W. J. Tompkins, "Detecting Ventricular Fibrillation," IEEE Engineering in Medicine and Biology, pp. 152-159, 1995.

[6] K. M. Ropella, J. M. Baerman, A. V. Sahakian, and S. Swiryn, "Differentiation of Ventricular Tachyarrhythmias," Circulation, vol. 82, pp. 2035-2043, 1990.

[7] S. Chen, N. V. Thakor, and M. M. Mower, "Analysis of Ventricular Arrhythmias: a Reliable Discrimination Technique," Computers in Cardiology 1986, pp. 179-182, 1986.

[8] A. Natarajan and N. V. Thakor, "A Sequential Hypothesis Testing Algorithm for Rapid Accurate Discrimination of Tachyarrhythmias," Proceedings of the Annual Conference on Engineering in Medicine and Biology, vol. 13, pp. 734-735, 1991.

[9] H. D. I. Abarbanel, Analysis of Observed Chaotic Data. New York, NY: Springer-Verlag New York, Inc., 1995.

[10] R. J. Povinelli, Time Series Data Mining: Identifying Temporal Patterns for Characterization and Prediction of Time Series Events, Dissertation, Marquette University, 1999.

[11] H. Bettermann and P. VanLeeuwen, "Evidence of Phase Transitions in Heart Period Dynamics," Biological Cybernetics, vol. 78, pp. 63-70, 1998.

[12] H. J. L. Marriott, Practical Electrocardiography, 7th ed. Baltimore, MD: Williams \& Wilkins, 1983.

[13] A. E. Norman, 12 Lead ECG Interpretation: A self-Teaching Manual. New York, NY: McGraw-Hill, Inc., 1992.

[14] R. M. Berne and M. N. Levy, Physiology, 3rd ed. Chicago, IL: Mosby Year Book, 1993.

[15] E. Braunwald, Heart Disease. A Textbook of Cardiovascular Medicine, 3 ed. Philadelphia: WB Saunders, 1988.

[16] J. L. Jones, "Ventricular Fibrillation," in Implantable Cardioverter-Defibrillator, I. Singer, Ed. Amonk, NY: Futura Publishing Company, Inc., 1994, pp. 43-67.

[17] W. H. Olson, "Tachyarrhythmia Sensing and Detection," in Implantable CardioverterDefibrillator, I. Singer, Ed. Amonk, NY: Futura Publishing Company, Inc., 1994, pp. 1342.

[18] I. Singer, "Ventricular Tachycardia," in Implantable Cardioverter-Defibrillator, I. Singer, Ed. Amonk, NY: Futura Publishing Company, Inc., 1994, pp. 13-42.

[19] T. M. Mitchell, Machine Learning. Madison, WI: WCB McGraw-Hill, 1997.

[20] X.-S. Zhang, Y.-S. Zhu, N. V. Thakor, and Z.-Z. Wang, "Detecting Ventricular Tachycardia and Fibrillation by Complexity Measure," IEEE Transactions on Biomedical Engineering, vol. 46, pp. 548-555, 1999.

[21] D. P. Zipes, E. N. Prystowsky, W. M. Miles, and J. J. Heger, "Future Directions: Electrical Therapy for Cardiac Tachyarrhythmias," PACE, vol. 7, pp. 606-610, 1984. 
[22] R. A. Winkle, "Electronic Control of Ventricular Tachyarrhythmias: Overview and Future Directions," PACE, vol. 7, pp. 1375-1379, 1984.

[23] A. S. Manolis, H. Rastegar, and N. A. M. Estes, "Automatic Implantable Cardioverter Defibrillator: Current Status," JAMA, vol. 262, pp. 1362-1368, 1989.

[24] E. Ott, Chaos in Dynamical Systems. New York, NY: Cambridge University Press, 1993. 